\title{
Mapping of S4 Over the Arabian Peninsula During Solar Minimum
}

This paper was downloaded from TechRxiv (https://www.techrxiv.org).

\section{LICENSE}

CC BY 4.0

SUBMISSION DATE / POSTED DATE

$26-09-2021 / 12-10-2021$

CITATION

Darya, Abdollah; Shaikh, Muhammad; Fernini, Ilias; AINaimiy, Hamid (2021): Mapping of S4 Over the Arabian Peninsula During Solar Minimum. TechRxiv. Preprint. https://doi.org/10.36227/techrxiv.16681885.v2

DOI

10.36227/techrxiv.16681885.v2 


\title{
Mapping of S4 Over the Arabian Peninsula During Solar Minimum
}

\author{
Abdollah Masoud Darya, Member, IEEE, Muhammad Mubasshir Shaikh, Ilias Fernini, and Hamid AlNaimiy
}

\begin{abstract}
In this letter, we study the temporal and spatial variability of ionospheric irregularities by generating high-resolution maps of the observed amplitude scintillation index (S4) using data from a multi-constellation and multi-frequency GNSS receiver. The study region is the Arabian Peninsula, which falls under the northern crest of the equatorial ionization anomaly (EIA). Even though the study was conducted during a solar minimum period, considerable occurrences of pre-sunset scintillation have been observed between 15-17 local time, particularly during the winter solstices. While most scintillation occurrences have been observed at low elevation (15 to 30 degrees), a considerable number of scintillation patches have been observed towards the north, east, and southeast of the receiver location, for elevation angles ranging from 40 to 60 degrees. Our analysis shows that BeiDou geostationary orbit (GEO) and inclined GEO (IGSO) satellites may have been the main contributor to the increased number of scintillation occurrences observed around the eastern side of the receiver as compared to the western side. Out of all the GNSS constellations with MEO satellites, GPS was the most impacted by amplitude scintillation, while BeiDou and Galileo satellites were the least affected. It is anticipated that the patches of ionospheric irregularities reported in this work would be further enhanced as the solar activity increases in the coming years. Therefore, this work can serve as a reference for future studies during periods of increased geomagnetic activity.
\end{abstract}

\section{INTRODUCTION}

$\mathbf{T}$ HE presence of ionospheric irregularities severely affects the quality of service of global navigation satellite systems (GNSS). These irregularities are typically caused by heightened geomagnetic activity and induce frequent fluctuations in signal intensity. The fluctuations in the signal intensity are referred to as amplitude scintillation [1]. The occurrence of ionospheric scintillation depends on various spatial and temporal factors, including time of day, season, and geographical location [2]. Ionospheric irregularities may be detected by continuously monitoring GNSS signals using ground-based receivers, or by radars, such as the ionosonde or multi-static high-frequency radars [3]-[5].

In [4], the authors used GNSS and ionosonde data to detect large-scale ionospheric irregularities. They generated maps that detected ionospheric irregularities originating from the equator at low and mid-latitude stations. In [5], the authors detected daytime ionospheric irregularities in the $\mathrm{E}$ and lower $\mathrm{F}$ regions around midday using a multi-static high-frequency radar. This is significant as ionospheric irregularities primarily occur during the post-sunset to midnight period.

The authors are with the Sharjah Academy for Astronomy, Space Sciences and Technology, University of Sharjah, UAE (e-mail: adarya@sharjah.ac.ae; mshaikh@sharjah.ac.ae; ifernini@sharjah.ac.ae; alnaimiy@sharjah.ac.ae).
The authors in [6] generated 5-minute amplitude scintillation index (S4) maps for India during a severe geomagnetic storm. The maps were generated using the Kriging method and had a resolution of $2^{\circ}$ by $2^{\circ}$. These maps successfully captured the features of the equatorial ionization anomaly over the Indian region, which is a neighboring region to the one considered in this letter. Following a similar methodology, the authors in [7] generated 30-minute S4 maps over southern China for a high solar activity period. The Kriging method had been used in both cases to interpolate for missing values. However, in this letter, we rely purely on data retrieved from observations in our work, and no attempt to interpolate for missing data was made. This should allow for a more accurate representation of the local ionosphere, particularly as we generate higher resolution maps as compared to previous work [6], [7]. Additionally, both [6], [7] generated maps for high solar activity periods and use only GPS data.

In this letter, we study the spatial and temporal variations of amplitude scintillation during a solar minimum period. The data was obtained from a multi-constellation and multifrequency GNSS receiver with scintillation monitoring capabilities (Septentrio PolaRx5S). The study region is the Arabian Peninsula $\left(25.2827^{\circ} \mathrm{N}, 55.4621^{\circ} \mathrm{E}\right)$, which falls under the northern crest of the equatorial ionization anomaly. The presence of pre-sunset scintillation for the Arabian peninsula region has been previously highlighted in [8], [9]. This is a feature that has previously been only observed in equatorial regions [10]. In this letter, we expand on the analysis performed in previous work by generating maps with a resolution of $0.2^{\circ}$ latitude and $0.5^{\circ}$ longitude to study the spatial distribution of the observed S4. Thus, our main objective is to identify the regions around the Arabian peninsula that are consistently influenced by the presence of amplitude scintillation on GNSS signals. We also compare the S4 observed on different orbits of the BeiDou GNSS constellation, and observations from the GPS, GLONASS, and Galileo constellations. As this study was conducted during a solar minimum, this work can serve as a reference for future studies during periods of increased geomagnetic activity.

The outline of this work is as follows. First, in section II, the methodology and techniques used in this letter are presented. Then, the results and discussion are given in section III. Finally, the conclusions and future work are in section IV.

\section{DAtA AND Methodology}

Amplitude scintillation is typically observed through the $\mathrm{S} 4$ index. The total $\mathrm{S} 4$ index $\left(\mathrm{S}_{\text {total }}\right)$ can be defined as 
TABLE I

THE SIGNALS OF THE FOUR DIFFERENT CONSTELLATIONS CONSIDERED IN THIS LETTER.

\begin{tabular}{|l|c|c|c|}
\hline & Signal 1 & Signal 2 & Signal 3 \\
\hline GPS & L1CA & L2C & L5 \\
\hline GLONASS & L1CA & L2C & - \\
\hline Galileo & L1BC & E5a & E5b \\
\hline BeiDou & B1 & B2 & B3 \\
\hline
\end{tabular}

the standard deviation of the $50 \mathrm{~Hz}$ signal intensity $(S I)$ normalized to the average signal intensity over 60 seconds. The corrected $\mathrm{S} 4$ index eliminates ambient noise $\left(\mathrm{S} 4_{\text {noise }}\right)$ from the total S4 index and is represented as [11]:

$$
\begin{aligned}
& \mathrm{S} 4=\sqrt{\mathrm{S}_{\text {total }_{1}}-\mathrm{S} 4_{\text {noise }}} \\
& \mathrm{S} 4=\sqrt{\frac{\left(S I^{2}\right)-(S I)^{2}}{(S I)^{2}}-\frac{100}{S N R}\left[1+\frac{500}{19 S N R}\right]}
\end{aligned}
$$

where $S N R$ is the signal-to-noise ratio of the signal. Note that for some instances, particularly for low total S4 values, the corrected S4 value was negative. Therefore, these values were set to zero.

This work utilizes S4 data generated by a PolaRx5S multiconstellation and multi-frequency GNSS receiver at the Sharjah Academy for Astronomy, Space Sciences and Technology $\left(25.2827^{\circ} \mathrm{N}, 55.4621^{\circ} \mathrm{E}\right)$, Sharjah, United Arab Emirates. The location is of particular interest as it falls within the northern crest of the equatorial ionization anomaly (EIA). In this work, S4 data from three different signals is obtained from each of the GPS, Galileo, and BeiDou constellations, while only two signals are observed from the GLONASS constellation (see Table I). While the space segments of GPS, GLONASS, and Galileo, consist exclusively of satellites orbiting at mediumearth-orbit (MEO), BeiDou combines satellites at MEO, geostationary orbits (GEO) and, inclined geostationary orbits (IGSO) [12]. By combining observations from these different constellations, a wider coverage area can be obtained.

The study period includes the summer, winter, and equinox seasons of 2019 and 2020, as well as the winter solstice of 2018 and the vernal equinox and summer solstice of 2021. Each season is taken as three months centered around their respective start dates, and the data for the equinoxes of 2019 and 2020 have been combined.

To map the observed S4 value to a particular geographical location, we use the thin shell ionospheric model (TSM) simplification [13]. We consider a perfectly spherical Earth with a radius equal to $6371 \mathrm{~km}$, and the TSM to be $350 \mathrm{~km}$ above the surface of the Earth [13].

Once the S4 values have been mapped to a geographical location, we divide the regional map ranging from 22 to $28^{\circ}$ latitude with a resolution of $0.2^{\circ}$, and from 45 to $65^{\circ}$ with a resolution of $0.5^{\circ}$. Thus each pixel would be of size $0.2^{\circ}$ latitude and $0.5^{\circ}$ longitude. Then the $\mathrm{S} 4$ values of 5 minutes over 90 days centered around the start date of each season are projected onto the map, and the mean S4 value for each pixel is taken by considering all observations that fall within the pixel during the specified time. Data from these 5-minute maps will be used in the following figures. Considering that the period analyzed is that of a solar minimum, three different categories for amplitude scintillation have been used, weak scintillation corresponding to $0.07 \leq S 4<0.2$, moderate scintillation corresponding to $0.2 \leq S 4<0.3$, and high scintillation corresponding to $S 4 \geq 0.3$ [9].

\section{RESULTS AND DISCUSSION}

Fig. 1 presents the seasonal trends based on the total number of occurrences of S4>0.07. The number of occurrences was obtained from the 5-minute maps for each season respectively. The black, blue, and red lines correspond to signals 1, 2, and 3 respectively (see Table I). Clearly, the equinoxes show more occurrences in general than the winter and summer solstices, particularly the equinox of 2020.

The most noticeable feature that can be seen in Fig. 1 is the enhancement in S4 values around 15 to 17 local time (LT), particularly during the winter solstices. This peak reached its maximum point at $16 \mathrm{LT}$ and was more prominent during 2018 and 2020. It may be attributed to the winter anomaly [14], where the ionization level during the winter season is higher than that of the summer season. This feature was previously reported in [8], [9], and during the winter solstices of the last solar cycle minimum in an equatorial location [10]. It is important to note that, unlike previous works, no enhancement of S4 was observed during the post-sunset to midnight (17-24 LT) period [7], [10]. Based on these observations, the winter solstices were chosen to be the focus of this work, with a particular focus on 15-17 LT.

Fig. 2 shows the signal 1 S4 maps for the 2018, 2019, and 2020 winter solstices around 15-17 LT. Each subplot represents 30 minutes, starting from 15 LT. Each subplot was obtained by overlapping 5-minute S4 observations within the same duration and taking the maximum. The intensity of the S4 values can be seen to increase from $15 \mathrm{LT}$, peaking around 16 LT, then gradually decreasing. The majority of high and moderate scintillation occurrences can be seen around the edges of the map. During 2018, S4 observation from the eastern and western edges of the map showed considerable amounts of ionospheric irregularities, from $45^{\circ}-50^{\circ}$, and $60^{\circ}-65^{\circ}$ longitude. However, during 2019 and 2020, the eastern edge $\left(60^{\circ}-65^{\circ}\right.$ longitude) was considerably more active than the west. Furthermore, the presence of a consistent patch that induced high scintillation was observed around the northeastern $\left(27^{\circ}-28^{\circ}\right.$ latitude and $60^{\circ}-65^{\circ}$ longitude $)$ edge of the map during all three years. Moderate and high S4 values were also observed around the receiver location, mostly through the 2018 and 2020 winter solstices from 15:30 LT to 16:30 LT. During this period, most pixels within the map can be seen to exceed an S4 value of 0.1. This is very significant for a solar minimum period. We note that these maps are a result of averaged S4 over 5 minutes for 90 days. This means that the S4 activity seen here was persistent for the entire season, i.e., over a total period of 90 days.

To better understand the data qualitatively from the receiver's viewpoint, and provide a different perspective on the spatial distribution of S4 values, we present the polar plot in Fig. 3. Similar to Fig. 2, Fig. 3 presents signal 1 S4 


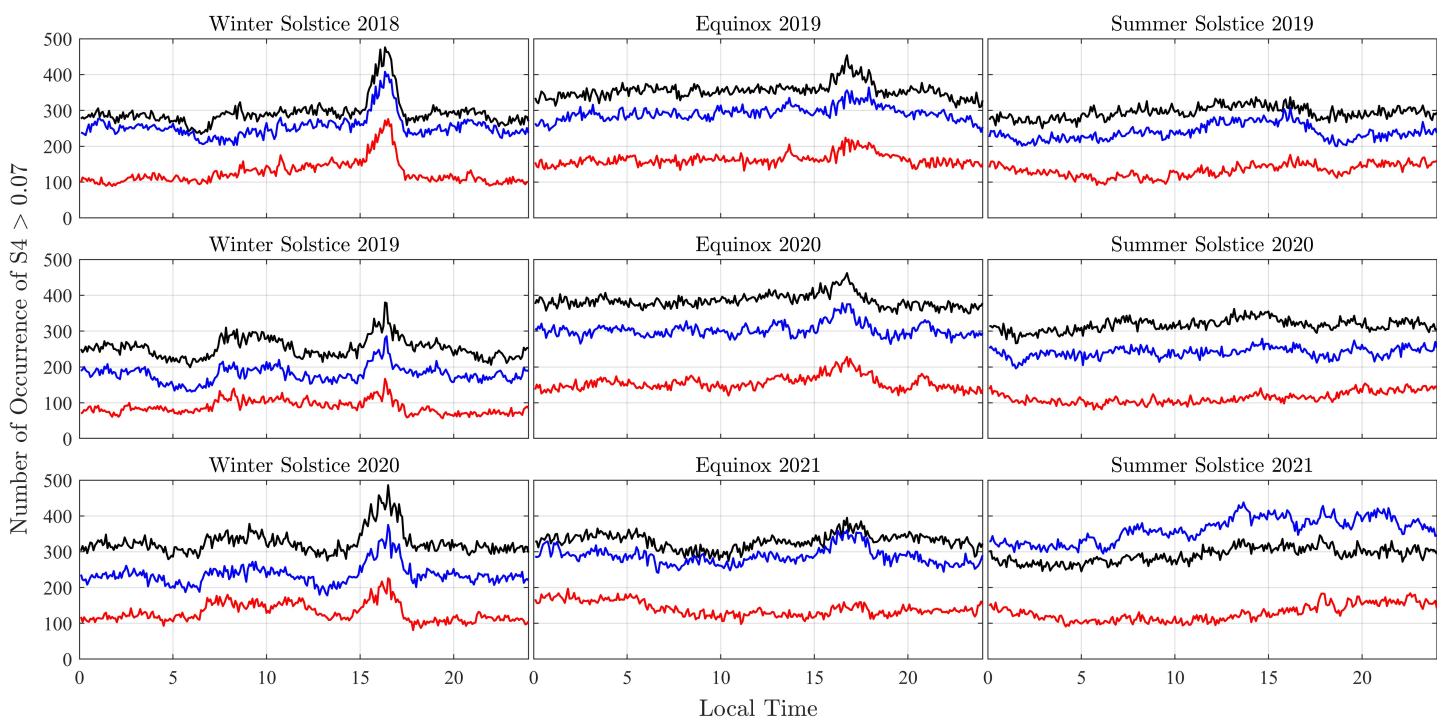

Fig. 1. Seasonal S4 > 0.07 occurrence trends. The black line represents signal 1, while the blue and red lines represent signals 2 and 3 respectively.

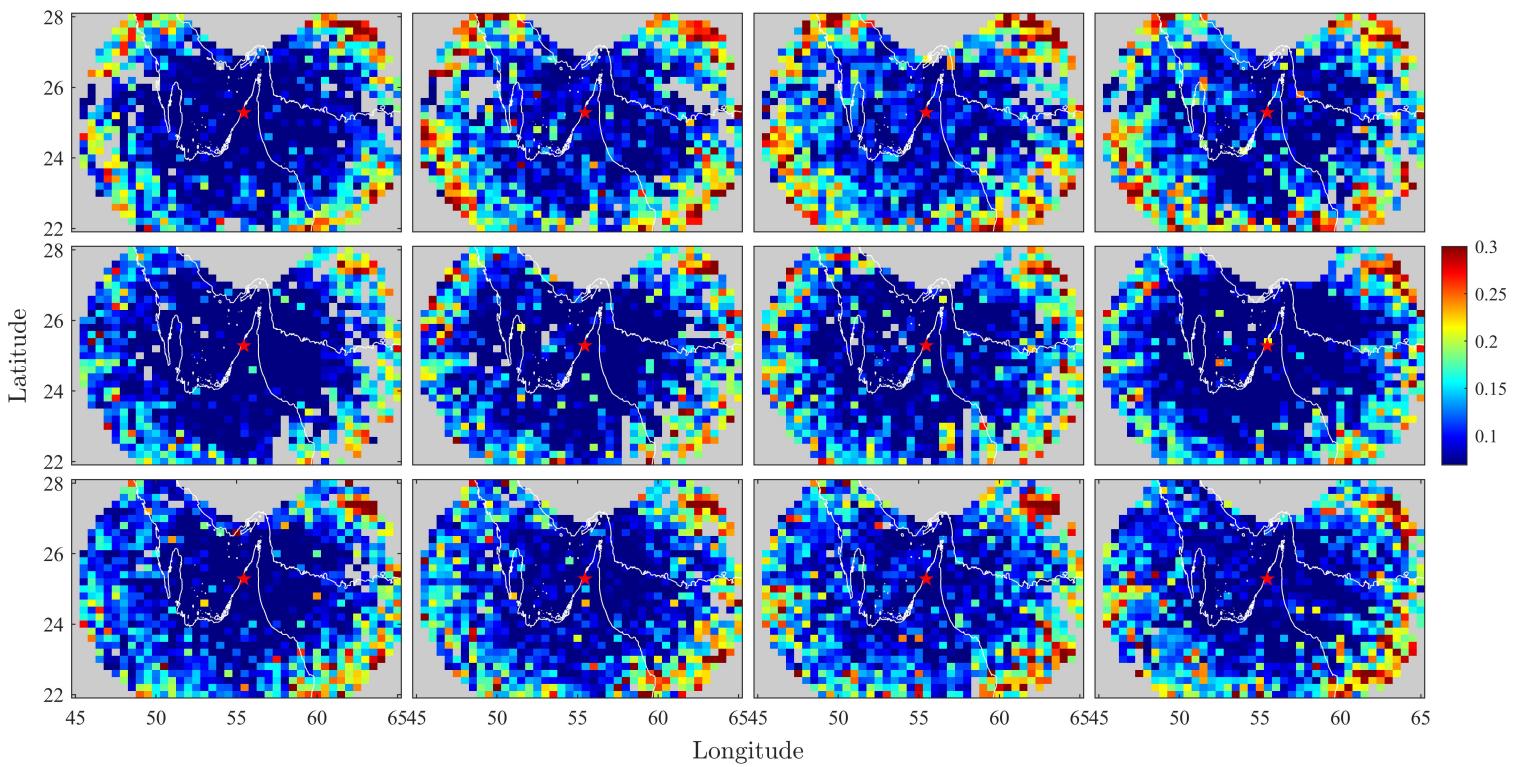

Fig. 2. The 30-minute S4 maps during 15-17 LT for the winter solstices of 2018 (first row), 2019 (second row), and 2020 (third row). The data in each map corresponds to the maximum of 6 consecutive 5-minute maps, for a total of 30 minutes. The color bars represent the $\mathrm{S} 4$ value ranging from $\leq 0.07$ (dark blue) to $\geq 0.3$ (dark red). Missing data pixels are represented as gray, the geographical features are outlined in white, and the receiver is represented as a red star.

data for the winter solstices of the study period from 1517 LT for 90 days. However, the data is not represented in terms of the IPP location, instead, the azimuth and elevation angles between the receiver and satellites were considered. The polar map has been divided into cells of size $30^{\circ}$ azimuth and $5^{\circ}$ elevation. The number of occurrences of $\mathrm{S} 4$ based on the S4 intensity levels has been counted and divided by the total number of occurrences for the entire slice of $30^{\circ}$ azimuth to produce the percentage occurrence. This should help us independently analyze each $30^{\circ}$ azimuth segment, and understand the relationship between azimuth, elevation, and S4 activity.

The majority of weak S4 occurrence can be seen at $15^{\circ}$ $30^{\circ}$ elevation during all three years. This is particularly the case toward the eastern part of the map for 2019 and 2020, where the occurrence ranged from $30 \%$ to $50 \%$, from $15^{\circ}$ to $20^{\circ}$ elevation. This corroborates the observations made in Fig. 2. For moderate and high scintillation, $40 \%-90 \%$ of the occurrences can be seen at $15^{\circ}-20^{\circ}$ elevation for all directions except north, where most occurrences range from $0 \%-40 \%$. This can be attributed to the data gap seen towards the north in Fig. 2. A particular area of interest is towards the southwest $\left(180^{\circ}-210^{\circ}\right.$ azimuth), where $70 \%-90 \%$ of occurrences of moderate to high scintillation have been observed at $15^{\circ}-20^{\circ}$ elevation. A patch of considerable occurrences of weak and moderate scintillation can be seen towards the north $\left(330^{\circ}\right.$ - $30^{\circ}$ azimuth) ranging between $45^{\circ}-60^{\circ}$ elevation for all years, but more prominent in terms of coverage during 2019 


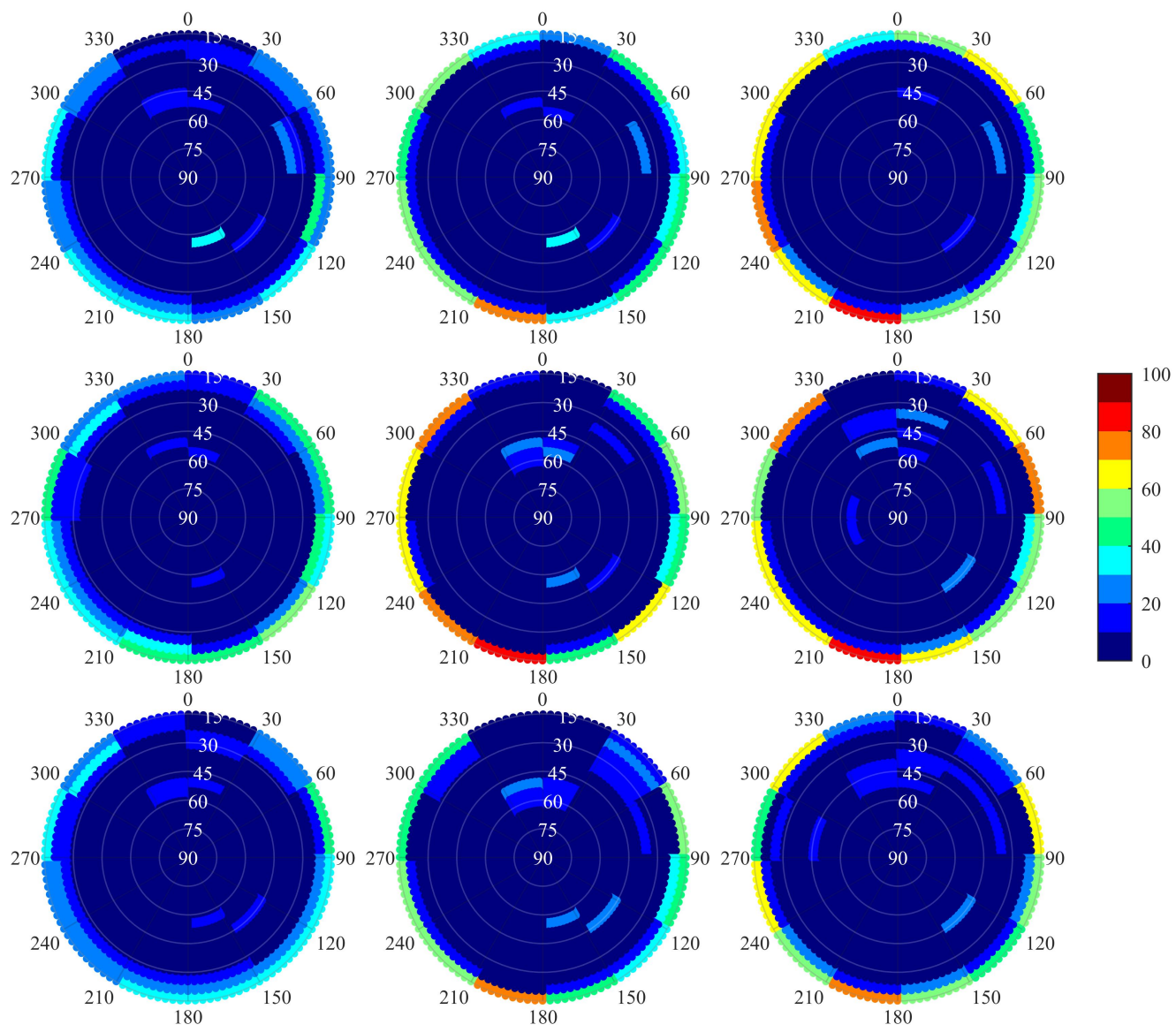

Fig. 3. The S4 polar plots (in terms of azimuth and elevation) during 15-17 LT for the winter solstices of 2018 (top row), 2019 (middle row), and 2020 (bottom row). The columns correspond to (from the left) $0.07 \leq \mathrm{S} 4<0.2$ (weak scintillation), $0.2 \leq \mathrm{S} 4<0.3$ (moderate scintillation), and $\mathrm{S} 4>0.3$ (high scintillation), respectively. The color bar corresponds to the percentage of occurrences. This percentage is found by dividing the number of occurrences in each cell by the total occurrences per 30 degrees azimuth.

and 2020. For high scintillation, the patch increases in area, ranging from $30^{\circ}-60^{\circ}$ elevation, more prominent during 2019 and 2020, and non-existent during 2018. During 2020, this patch also extends towards the east. An additional feature that can be seen is the existence of a small patch ranging between $45^{\circ}-60^{\circ}$ elevation, towards the south to southeast direction $\left(120^{\circ}-180^{\circ}\right.$ azimuth), with scintillation occurrence from $10 \%$ to $40 \%$. For all years, the occurrence of scintillation towards the west to south direction $\left(180^{\circ}-300^{\circ}\right.$ azimuth $)$ at mid $\left(30^{\circ}\right.$ $-60^{\circ}$ ) elevation is minimal, ranging from $0 \%-10 \%$, unlike the east, where various patches of $10 \%-30 \%$ occurrence can be seen. This is also consistent with what was observed in Fig. 2 . The only occurrence of high scintillation above $0 \%-10 \%$ for high $(60 \%-90 \%)$ elevation was in 2019 towards the west $\left(240^{\circ}-300^{\circ}\right.$ azimuth).

To better understand the amplitude scintillation observed by each constellation separately, we present Fig. 4. In the left panel of Fig. 4, the winter solstice peak around 15-17 LT is observed from all satellite constellations and signals, more prominent for GPS, Galileo, and BeiDou (BD), and less so for GLONASS, where several peaks at different times are observed. Expanding on the analysis of Fig. 1, most of the occurrences can be seen from signal 1 of GPS, followed by signals 1 and 2 from GLONASS. For GLONASS, both signals seem to have similar numbers of occurrences, a feature that is also shared by signals 2 and 3 from Galileo.

While the space segment of the four major GNSS constellations consists mainly of MEO satellites, BeiDou incorporates satellites in the GEO and IGSO as well. The bar chart in Fig. 4 presents the percentage occurrence of amplitude scintillation on signal 1 for the winter solstices of 2018,2019, and 2020 at 15-17 LT. The BeiDou IGSO and MEO satellites, as well as the Galileo MEO satellites, displayed similar percentage occurrences. On the other hand, the GPS satellites had the maximum percentage occurrence for all scintillation categories, followed by GLONASS. The three BeiDou GEO satellites had the minimum percentage occurrence. This can be attributed to their higher elevation visibility over the Arabian peninsula (see the polar plot in Fig. 4). From the polar plot in Fig. 4, we can deduce that the patches of ionospheric irregularities seen towards the north in Fig. 3 could not have resulted from the GEO and IGSO BeiDou satellites, and instead were fully based on MEO satellite observations. Furthermore, the increased intensity of scintillation towards the eastern and southeastern directions as observed in Fig. 2 and Fig. 3 could have been induced by the BeiDou GEO and IGSO satellites as these irregularities were not present on the western side of the maps at such high elevation. These observations from 


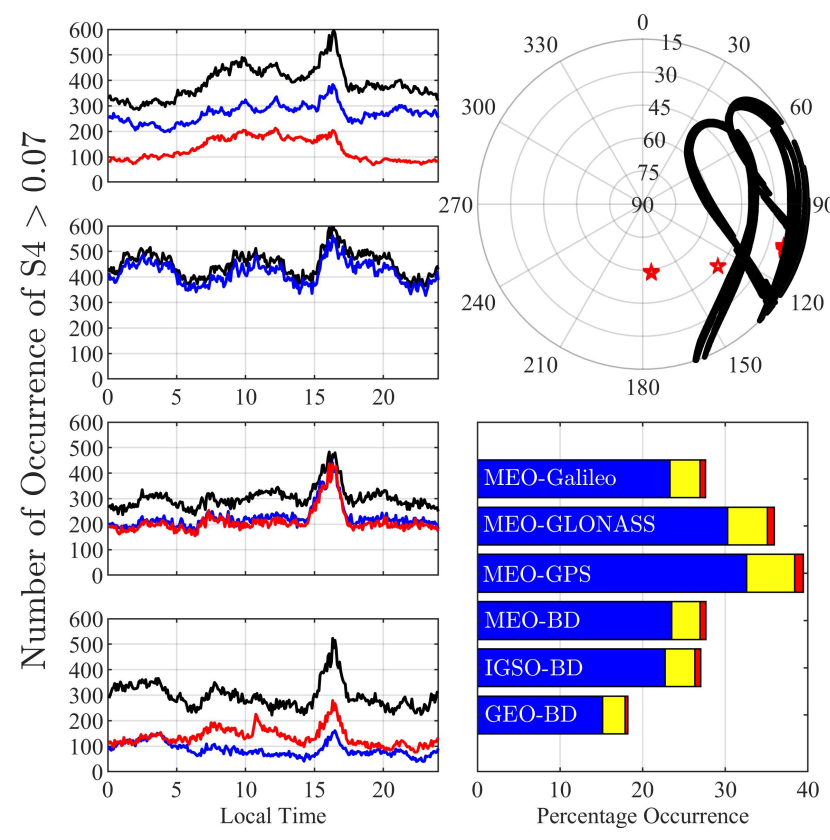

Fig. 4. Left Panel: The number of occurrences of S4 $>0.07$ versus local time for the combined data of the winter solstices of 2018, 2019, and 2020 for GPS (first row), GLONASS (second row), Galileo (third row), and BeiDou (fourth row). The black line represents signal 1, while the blue and red lines represent signals 2 and 3 respectively. Top Right Panel: The polar plot of the azimuth and elevation coordinates of signal $1 \mathrm{~S} 4$ observations obtained from GEO (red) and IGSO (black) BeiDou satellites. Bottom Right Panel: Stacked bar chart representing the occurrences of signal $1 \mathrm{~S} 4$ for different orbits. The blue, yellow, and red bars represent weak, moderate, and high scintillation respectively.

Fig. 3 point towards an interesting feature of GEO and IGSO satellites as part of a GNSS system. Further investigation of the BeiDou GEO and IGSO satellites would provide more interesting features and confirm the observations presented in this work.

\section{Summary AND CONCLUSION}

In this letter, we have shown that ionospheric irregularities may be localized effectively through multi-constellation observations over a geographical region of interest. We studied the temporal and spatial variability of amplitude scintillation caused by ionospheric irregularities by generating highresolution S4 maps over the Arabian Peninsula region. These maps have been generated using data from four different GNSS constellations, and different signals for each constellation. Combining observations from different constellations enabled us to generate high-resolution maps of the possible regions of irregularities. The study period corresponded to a solar minimum period from the winter solstice of 2018 to the summer solstice of 2021. We may summarize the findings of this letter in the following points:

- We found the values of S4 peaking at 15-17 LT during the winter solstice, particularly during the 2018 and 2020 winter solstices, and to a lesser extent, during the equinoxes at the same time.

- The pre-sunset enhancement of S4 during the winter solstice was observed in greater effect in GPS, Galileo, and BeiDou signals, particularly for MEO satellites, and less so for GEO and IGSO BeiDou satellites.

- A patch of ionospheric scintillation occurrences has been observed in Fig. 3 towards the north of the GNSS station between $45^{\circ}-60^{\circ}$ elevation.

- More occurrences of scintillation towards the east of the receiver have been observed in Fig. 2 and Fig. 3 as compared to the western side. IGSO BeiDou satellites could have been the main contributor to these observations.

- Patches of ionospheric scintillation observed towards the south to southeast of the receiver in Fig. 3 may have been primarily caused by GEO BeiDou satellite observations.

It is anticipated that the patches of ionospheric irregularities reported in this work would be further enhanced as the solar activity increases in the coming years. The interesting observations presented by the BeiDou GEO and IGSO satellites would be further investigated in the future to understand the possible cause of enhanced scintillation in their coverage area.

\section{REFERENCES}

[1] C. Cristodaro, F. Dovis, N. Linty, and R. Romero, "Design of a configurable monitoring station for scintillations by means of a gnss software radio receiver," IEEE Geoscience and Remote Sensing Letters, vol. 15, no. 3, pp. 325-329, 2018.

[2] R. K. Crane, "Ionospheric scintillation," Proceedings of the IEEE, vol. 65, no. 2, pp. 180-199, 1977.

[3] W.-H. Yeh, C.-Y. Lin, J.-Y. Liu, S.-P. Chen, T.-Y. Hsiao, and C.Y. Huang, "Superposition property of the ionospheric scintillation s4 index," IEEE Geoscience and Remote Sensing Letters, vol. 17, no. 4, pp. 597-600, 2019.

[4] C. Jiang, L. Wei, G. Yang, E. Aa, T. Lan, T. Liu, J. Liu, and Z. Zhao, "Large-scale ionospheric irregularities detected by ionosonde and gnss receiver network," IEEE Geoscience and Remote Sensing Letters, vol. 18, no. 6, pp. 940-943, 2020.

[5] J. Wu, G. Yang, Y. Zhang, C. Zhou, and Z. Zhao, "Detection of daytime ionospheric irregularities at low latitudes with a multistatic hf radar," IEEE Geoscience and Remote Sensing Letters, vol. 16, no. 6, pp. 839843, 2019.

[6] P. B. S. Harsha, D. V. Ratnam, M. L. Nagasri, M. Sridhar, and K. P. Raju, "Kriging-based ionospheric tec, roti and amplitude scintillation index (s 4) maps for india," IET Radar, Sonar \& Navigation, vol. 14, no. 11 , pp. $1827-1836,2020$.

[7] W. Geng, W. Huang, G. Liu, E. Aa, S. Liu, Y. Chen, and B. Luo, "Generation of ionospheric scintillation maps over southern china based on kriging method," Advances in Space Research, vol. 65, no. 12, pp. 2808-2820, 2020.

[8] M. Shaikh, I. Fernini, G. Gopakumar, and N. Alameri, "Occurrence of pre-sunset 1-band scintillation due to strong presence of sporadic-e over arabian peninsula," Advances in Space Research, vol. 65, no. 10, pp. 2412-2423, 2020.

[9] M. M. Shaikh, G. Gopakumar, A. Hussein, A. Kashcheyev, and I. Fernini, "Daytime gnss scintillation due to es over arabian peninsula during low solar activity," Results in Physics, vol. 20, p. 103761, 2021.

[10] A. Seif, M. Abdullah, A. M. Hasbi, and Y. Zou, "Investigation of ionospheric scintillation at ukm station, malaysia during low solar activity," Acta Astronautica, vol. 81, no. 1, pp. 92-101, 2012.

[11] A. Van Dierendonck, J. Klobuchar, and Q. Hua, "Ionospheric scintillation monitoring using commercial single frequency c/a code receivers," in proceedings of ION GPS, vol. 93. Citeseer, 1993, pp. 1333-1342.

[12] Y. Yang, W. Gao, S. Guo, Y. Mao, and Y. Yang, "Introduction to beidou3 navigation satellite system," NAVIGATION, Journal of the Institute of Navigation, vol. 66, no. 1, pp. 7-18, 2019.

[13] P. Rama Rao, K. Niranjan, D. Prasad, S. Gopi Krishna, and G. Uma, "On the validity of the ionospheric pierce point (ipp) altitude of $350 \mathrm{~km}$ in the indian equatorial and low-latitude sector," in Annales Geophysicae, vol. 24, no. 8. Copernicus GmbH, 2006, pp. 2159-2168.

[14] F. Azpilicueta and B. Nava, "A different view of the ionospheric winter anomaly," Advances in Space Research, vol. 67, no. 1, pp. 150-162, 2021. 